\title{
Diagnostic value of amyloid-PET and tau-PET: a head-to-head comparison
}

\author{
Daniele Altomare ${ }^{1,2}$ (1) $\cdot$ Camilla Caprioglio ${ }^{1,2} \cdot$ Frédéric Assal $^{3,4} \cdot$ Gilles Allali $^{3,5} \cdot$ Aline Mendes $^{6} \cdot$ Federica Ribaldi $^{1,7,8}$. \\ Kelly Ceyzeriat ${ }^{9,10}$. Marta Martins ${ }^{1,2}$. Szymon Tomczyk ${ }^{1,2}$ - Sara Stampacchia ${ }^{9}$ • Alessandra Dodich ${ }^{11}$. \\ Marina Boccardi ${ }^{12} \cdot$ Christian Chicherio $^{2}$ - Giovanni B. Frisoni ${ }^{1,2} \cdot$ Valentina Garibotto $^{8,13}$
}

Received: 28 October 2020 / Accepted: 7 February 2021 / Published online: 27 February 2021

(C) The Author(s) 2021

\begin{abstract}
Purpose Assess the individual and combined diagnostic value of amyloid-PET and tau-PET in a memory clinic population. Methods Clinical reports of 136 patients were randomly assigned to two diagnostic pathways: AMY-TAU, amyloid-PET is presented before tau-PET; and TAU-AMY, tau-PET is presented before amyloid-PET. Two neurologists independently assessed all reports with a balanced randomized design, and expressed etiological diagnosis and diagnostic confidence (50-100\%) three times: (i) at baseline based on the routine diagnostic workup, (ii) after the first exam (amyloid-PET for the AMY-TAU pathway, and tau-PET for the TAU-AMY pathway), and (iii) after the remaining exam. The main outcomes were changes in diagnosis (from $\mathrm{AD}$ to non-AD or vice versa) and in diagnostic confidence.

Results Amyloid-PET and tau-PET, when presented as the first exam, resulted in a change of etiological diagnosis in $28 \%(p=$ $0.006)$ and $28 \%(p<0.001)$ of cases, and diagnostic confidence increased by $18 \%(p<0.001)$ and $19 \%(p<0.001)$ respectively, with no differences between exams $(p>0.05)$. We observed a stronger impact of a negative amyloid-PET versus a negative tauPET $(p=0.014)$. When added as the second exam, amyloid-PET and tau-PET resulted in a further change in etiological diagnosis in $6 \%(p=0.077)$ and $9 \%(p=0.149)$ of cases, and diagnostic confidence increased by $4 \%(p<0.001)$ and $5 \%(p<0.001)$ respectively, with no differences between exams $(p>0.05)$.

Conclusion Amyloid-PET and tau-PET significantly impacted diagnosis and diagnostic confidence in a similar way, although a negative amyloid-PET has a stronger impact on diagnosis than a negative tau-PET. Adding either of the two as second exam further improved diagnostic confidence.

Trial number PB 2016-01346.
\end{abstract}

Keywords Amyloid $\cdot$ Tau $\cdot$ PET $\cdot$ Florbetapir $\cdot$ Flutemetamol $\cdot$ Flortaucipir

This article is part of the Topical Collection on Translational research

Daniele Altomare

Daniele.Altomare@unige.ch

1 Laboratory of Neuroimaging of Aging (LANVIE), University of Geneva, Geneva, Switzerland

2 Memory Clinic, Geneva University Hospitals, Geneva, Switzerland

3 Division of Neurology, Department of Clinical Neurosciences, Geneva University Hospitals, Geneva, Switzerland

4 Faculty of Medicine, University of Geneva, Geneva, Switzerland

5 Department of Neurology, Division of Cognitive \& Motor Aging, Albert Einstein College of Medicine, Yeshiva University, Bronx, NY, USA

6 Division of Geriatrics, Department of Rehabilitation and Geriatrics, Geneva University Hospitals, Geneva, Switzerland
7 Laboratory of Alzheimer's Neuroimaging and Epidemiology (LANE), Saint John of God Clinical Research Centre, Brescia, Italy

8 Department of Molecular and Translational Medicine, University of Brescia, Brescia, Italy

9 Laboratory of Neuroimaging and Innovative Molecular Tracers (NIMTlab), Geneva University Neurocenter and Faculty of Medicine, University of Geneva, Geneva, Switzerland

10 Division of Adult Psychiatry, Department of Psychiatry, Geneva University Hospitals, Geneva, Switzerland

11 Center for Mind/Brain Sciences (CIMeC), University of Trento, Rovereto, Italy

12 Late Translational Dementia Research Group, German Center for Neurodegenerative Diseases (DZNE), Rostock-Greifswald site, Rostock, Germany

13 Division of Nuclear Medicine and Molecular Imaging, Geneva University Hospitals, Geneva, Switzerland 


\section{Introduction}

The pathological accumulation of aggregated proteins in the brain represents a hallmark of most neurodegenerative diseases. Specifically, amyloid and tau are the two main proteins involved in Alzheimer's disease (AD). AD pathophysiological changes precede clinical symptoms by about 20 years [1]. Therefore, the use of amyloid and tau biomarkers allows to identify $\mathrm{AD}$ in its early phases, i.e., in patients with mild cognitive impairment (MCI), and make differential diagnosis in cases with atypical presentations [1].

Thus, the recent development of PET tracers allowing to assess and quantify brain amyloid and tau deposition in vivo represented a breakthrough for research in this field. Indeed, such pivotal tools have been recently incorporated in research frameworks defining AD based only on the abnormal deposition of both proteins irrespective of the clinical profile $[2,3]$.

The first study on humans involving an amyloid-PET ligand was published 17 years ago [4], and converging evidence suggests that amyloid-PET is a helpful support for clinicians, leading to changes of diagnoses and treatment plan based on traditional work-up and increase of confidence on etiological diagnosis [5]. Tau-PET was more recently introduced [6], and 18F-Flortaucipir, the most widely used firstgeneration tau-PET tracer, has shown partial analytical validity and preliminary evidence of clinical validity [7] and has recently been approved by the FDA for estimating density and distribution of tau neurofibrillary tangles in vivo [8], but evidence on the diagnostic value of tau-PET is not available yet.

To date, evidence on the relative and combined diagnostic value of biomarkers assessing amyloid and tau is scanty. Recently, Ramusino and colleagues compared the relative incremental diagnostic value of amyloid-PET and CSF $\left(A \beta_{42}\right.$, p-tau, t-tau), showing that amyloid-PET induces greater changes in the diagnosis of AD patients and an overall increase of diagnostic confidence when performed both as the first investigation and even after CSF, possibly because of the higher negative predictive value of amyloid-PET [9]. However, no studies have assessed the diagnostic value of amyloid-PET and tau-PET (and their combination) in clinical practice.

This study aims to provide preliminary evidence on the diagnostic value, in terms of changes of etiological diagnosis and diagnostic confidence, of amyloid-PET and tauPET performed using 18F-Flortaucipir, both individually and in combination, in an unselected memory clinic population. In order to investigate the impact of amyloid-PET and tau-PET in patients with different cognitive stages, the analyses were replicated in subsamples including only patients with subjective cognitive decline (SCD), or only those with MCI, or only those with dementia. Our a priori hypothesis is that tau-PET has a greater diagnostic value than amyloid-PET.

\section{Material and methods}

\section{Participants}

The participants were 136 patients with cognitive complaints recruited consecutively and evaluated at the Geneva Memory Clinic, who underwent, from November 2015 to July 2020, a diagnostic workup including clinical and neuropsychological assessments, MRI, and amyloid-PET and tau-PET within an ongoing prospective research study. The study focused on the interplay between amyloidosis and tau-related neurodegeneration was approved by the local ethic committee (PB 201601346) and has been conducted in accordance with the principles of the Declaration of Helsinki and the International Conference on Harmonization Good Clinical Practice. Each subject provided a voluntary written informed consent for the participation in the study. The mean time interval between amyloid-PET and tau-PET is 5 months ( $\mathrm{SD}=5$ months).

\section{Study design}

Figure 1 illustrates the study design. To assess the relative incremental diagnostic value of amyloid-PET and tau-PET, the data of the study participants were retrospectively randomized into two study arms resulting in two distinct diagnostic pathways: (i) AMY-TAU, amyloid-PET followed by tau-PET; or (ii) TAU-AMY, tau-PET followed by amyloid-PET. Two independent raters (FA and GA, both neurologists with $\geq 15$ years of experience in the field of neurodegenerative disorders) blindly assessed patients' clinical reports three times with a balanced randomization of patients relative to the arm and the order of the two PET exams (Fig. 1). At T0 (baseline), the two raters received the anonymized clinical reports including clinical and neuropsychological assessments as well as the MRI report, and were asked to indicate a cognitive stage (e.g., subjective cognitive decline (SCD) [10], MCI [11], dementia [12]) and an etiological diagnosis and rate their diagnostic confidence (from $50 \%$, max uncertainty, to $100 \%$, max certainty) in such etiological diagnosis for each patient. At T1, the two raters were asked to revise the baseline etiological diagnosis and diagnostic confidence for each patient based on the first exam received (either amyloid-PET or tau-PET, according to the study design): for patients in ARM1, the first rater received the amyloid-PET report as the first exam, while the second rater received the tau-PET report; for patients in ARM2, the first rater received the tau-PET report as the first exam, while the second rater received the amyloid-PET report. At T2, the two raters could revise etiological diagnosis and diagnostic confidence one last time, based on the remaining exam.

Physicians made etiological diagnoses using conventional criteria. The etiological diagnoses were categorized into two categories: (i) $\mathrm{AD}$, including all the diagnoses involving $\mathrm{AD}$ (e.g., $\mathrm{AD}[11,12], \mathrm{AD}$ in comorbidity with cerebrovascular 


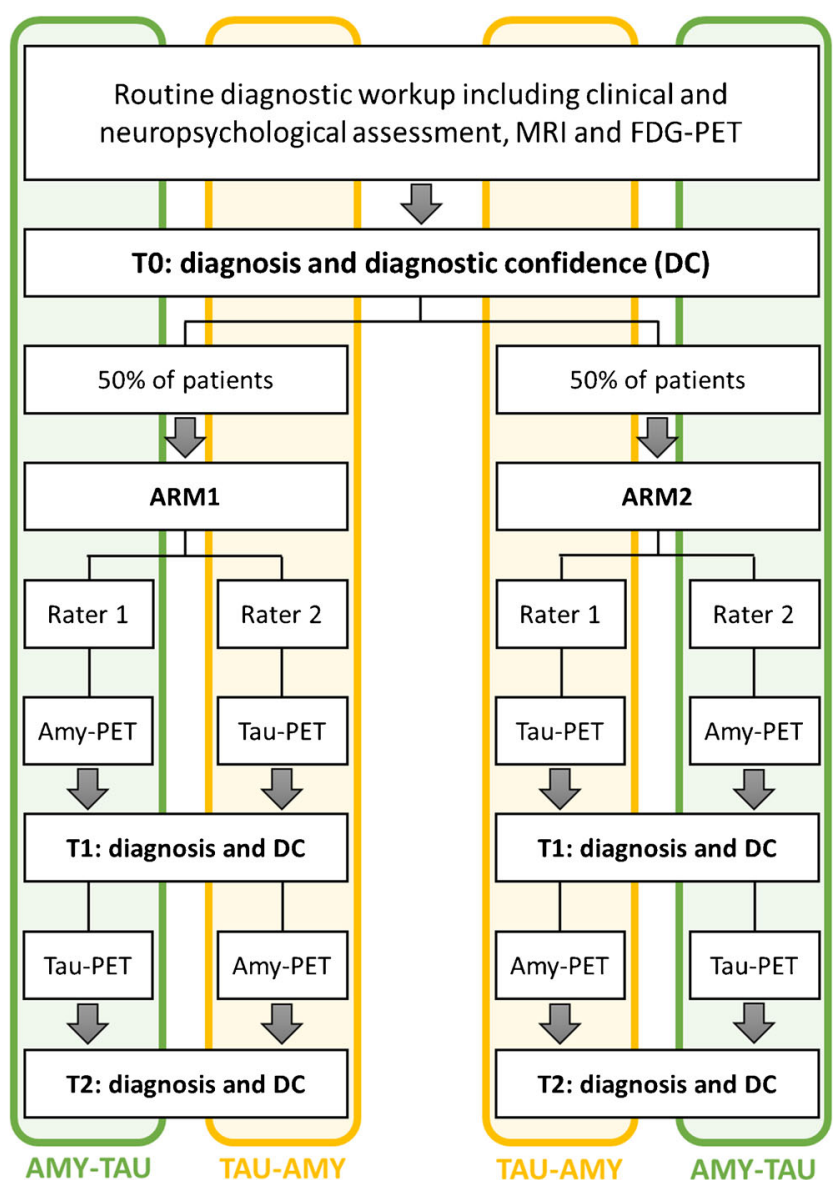

Fig. 1 Study design

disease (CVD), $\mathrm{AD}$ with frontotemporal lobar degeneration (FTLD), AD with dementia with Lewy bodies (DLB), AD with psychiatric disorders, and $\mathrm{AD}$ with alcohol abuse), and (ii) non- $\mathrm{AD}$, including all the remaining diagnoses without AD in comorbidity (e.g., CVD [13-15], FTLD [16], DLB [17], psychiatric disorders), suspected non-Alzheimer's disease (SNAP) $[18,19]$, and normal aging.

A third rater (AM) was asked to assess the cases discordant for cognitive stage or etiological diagnosis (AD or non-AD) at baseline. Finally, the two initial raters (FA and GA) were asked to revise those cases in which their diagnoses were in disagreement with the majority, i.e., the assessment of the other two raters.

\section{Outcomes}

The two primary study outcomes were change in the etiological diagnosis (from $\mathrm{AD}$ to non- $\mathrm{AD}$, or from non- $\mathrm{AD}$ to $\mathrm{AD}$ ) and change in diagnostic confidence across timepoints in the whole sample. Secondary outcomes included changes in diagnosis and diagnostic confidence according to the PET results. These analyses were replicated in each cognitive stage group (i.e., $\mathrm{SCD}, \mathrm{MCI}$, dementia).

\section{Amyloid-PET and tau-PET acquisition}

Amyloid-PET scanning using [18-F] radioligands was performed at the nuclear medicine and molecular imaging division at Geneva University Hospitals with a Siemens Biograph mCT PET scanner. Amyloid-PET images were acquired using 18F-Florbetapir $(n=72)$ or 18 F-Flutemetamol $(n=64)$ tracers. 18F-Florbetapir images were acquired $50 \mathrm{~min}$ after the intravenous administration of $200 \mathrm{Mbq}$ of the radiotracer ( $3 \times 5$-min image frames). 18F-Flutemetamol images were acquired $90 \mathrm{~min}$ after the intravenous administration of $150 \mathrm{MBq}$ of the radiotracer $(4 \times 5$-min image frames).

Tau-PET scans were acquired using 18F-Flortaucipir (18FAV1451), synthesized at the Center for Radiopharmaceutical Sciences ETH-PSI-USZ in Zurich, Switzerland, under license from the IP owner (Avid/Lilly). Image acquisition was performed 75 min after injection of $180 \mathrm{MBq}$ of $18 \mathrm{Fflortaucipir}$ (6 $\times 5$-min frames) [20].

\section{Amyloid-PET and tau-PET assessment}

Amyloid positivity was determined in each patient by an expert in nuclear medicine $(\mathrm{VG},>15$ years of experience in the field) using visual assessment and standard operating procedures approved by the European Medicines Agency [21, 22].

Tau distribution was determined in each patient by an expert in nuclear medicine $(\mathrm{VG})$, who visually analyzed images in agreement with recently published recommendation [23], describing regions of increased 18F-Flortaucipir uptake with respect to Braak stages [24]. Further details on the visual assessment of tau-PET scans are available in Supplementary Section 2.5 in the Supplementary Material. Raters had access to PET reports. To binarize the tau-PET result in data analyses, Braak stages 0-III were considered as tau negative, and Braak stages IV-VI as tau positive, in accordance with current knowledge on the cognitive impact of tau pathology [25] and on the detectability of tau pathology with 18F-Flortaucipir [26], and consistently with a recent study [27].

\section{Statistical methods}

Randomization into study arms was performed using a complete random assignment procedure.

Values are expressed as median $\left(25^{\text {th }}-75^{\text {th }}\right.$ percentile $)$ for continuous variables, and percentages (raw number) for the categorical variables. Sociodemographic and clinical features in ARM1 and ARM2 were compared using the MannWhitney test for continuous variables, and proportion test for categorical variables.

The inter-rater agreement between the blind raters for the clustered etiologic diagnoses (AD and non- $\mathrm{AD})$ at the different timepoints was assessed using the unweighted Cohen's $k$ coefficient, and strength of agreement classified as slight $(0.00-0.20)$, 
fair $(0.20-0.40)$, moderate $(0.40-0.60)$, good $(0.60-0.80)$, and very good $(>0.80)$ with $95 \%$ confidence intervals $(\mathrm{CI})$. For the inter-rater agreement at baseline, we used the first assessment of the two raters (i.e., before they aligned the discordant cases with the third rater's assessment). The non-overlap between Cohen's $k$ 95\% CI was considered as statistically significant.

The McNemar chi-squared test $\left(\chi^{2}\right)$ was used to assess changes in diagnosis (from $\mathrm{AD}$ to non- $\mathrm{AD}$ or from non- $\mathrm{AD}$ to $\mathrm{AD}$ ) after PET scans. Proportion test $\left(\chi^{2}\right)$ was used to assess the difference in diagnostic changes between pathways.

Between-pathway differences in diagnostic confidence at each timepoint and changes in diagnostic confidence along timepoints within each pathway were assessed using a linear mixed model with three repeated factors (timepoint: T1 and T2; rater: rater 1 and rater 2; pathway: AMY-TAU and TAUAMY) and subjects as random effects; the dependent variable was the difference in diagnostic confidence between $\mathrm{T} 1$ and $\mathrm{T} 0$, and $\mathrm{T} 2$ and $\mathrm{T} 0$. We assessed the estimated marginal means of the interaction between pathway and timepoint using the Bonferroni correction to adjust $p$ values of the pairwise comparisons.

All statistical analyses were performed with $R$, version 4.0.2 (R Foundation for statistical computing, https://www.r-project.org/).

\section{Results}

\section{Participants' features}

A total of 136 patients were involved in this study. Table 1 illustrates their demographic and clinical features. Patients were on average $74(70-78)$ (median, $25^{\text {th }}-75^{\text {th }}$ percentile) years old, males and females were equally distributed. Among them, 51\% (69/136) had MCI, 32\% (43/136) had dementia, and 18\% (24/136) had SCD. As a result of the randomization, there were no significant differences in demographic and clinical features between ARM1 and ARM2 patients (Table 1). In SCD patients with a diagnosis other than "normal aging," the highest baseline diagnostic confidence was $85 \%$, and dropped to $55 \%$ when focusing only on those $(n=3)$ with a baseline etiological diagnosis of AD (both values are below the threshold conventionally used to rate diagnostic confidence as "very high," i.e., 90\%). [28]

The baseline etiological diagnosis was inconsistent with the amyloid-PET result in 28\% (38/136) of cases (i.e., 28 amyloid-negative $\mathrm{AD}$, and 10 amyloid-positive non-AD patients), and with the tau-PET result in $37 \%$ (51/136) of cases (i.e., 46 tau-negative $\mathrm{AD}$, and 5 tau-positive non- $\mathrm{AD}$ patients).

\section{Concordance between raters}

The inter-rater agreement on the etiological diagnosis ( $\mathrm{AD}$ or non-AD) was fair at baseline (71\% concordance rate, unweighted $k=0.36,95 \%$ CI $0.20-0.52$ ), but increased to good with biomarker availability at T1 $(87 \%$ concordance rate, unweighted $k=0.74,95 \%$ CI $0.62-0.85)$ and T2 (88\% concordance rate, unweighted $k=0.76,95 \%$ CI $0.66-0.87$ ).

\section{Change in diagnosis}

In the AMY-TAU pathway, 28\% (38/136; $\left.\chi^{2}=7.6, p=0.006\right)$ of patients' diagnoses changed after amyloid-PET when presented as the first exam (T1), and an additional 9\% (12/136; $\chi^{2}=2.1, p=0.149$ ) of cases showed a further change after tauPET as the second exam (T2) (Table 2). In the TAU-AMY pathway, 28\% (38/136; $\left.\chi^{2}=25.3, p<0.001\right)$ of patients' diagnoses changed after tau-PET when presented as the first exam (T1), and an additional $6 \%\left(8 / 136 ; \chi^{2}=3.1, p=0.077\right)$ of cases showed a further change after amyloid-PET as the second exam (T2) (Table 2). These changes in diagnosis were not statistically different between the two pathways both at $\mathrm{T} 1\left(\chi^{2}=0, p=\right.$ $1.00)$ and $\mathrm{T} 2\left(\chi^{2}=0.5, p=0.486\right)$.

All but one case of changes in diagnosis were observed when the PET results were inconsistent with the previous diagnosis (i.e., negative PET in AD patients, or positive PET in non-AD patients). Figure 2 illustrates how the diagnoses changed in the cases with PET results inconsistent with the previous diagnosis. In patients with a baseline diagnosis of $\mathrm{AD}$ and an inconsistent PET scan, $100 \%$ (28/28) changed their diagnosis to non-AD after a negative amyloid-PET, and 76\% (35/46) changed to non-AD after a negative tau-PET, denoting a statistically stronger impact of a negative amyloid-PET versus a negative tau-PET $\left(\chi^{2}=6.1\right.$, $p=0.014)$. In patients with a baseline diagnosis of non- $\mathrm{AD}$ and an inconsistent PET scan, 100\% (10/10) changed their diagnosis to $\mathrm{AD}$ after a positive amyloid-PET, and $60 \%$ (3/5) changed to $\mathrm{AD}$ after a positive tau-PET (the two patients with confirmed non-AD diagnosis after positive tau-PET were both diagnosed as FTLD at baseline), a non-significantly different impact $\left(\chi^{2}=\right.$ $1.8, p=0.179)$ possibly due to small sample size. In patients with an etiological diagnosis of $\mathrm{AD}$ already supported by a first PET scan, a further change in diagnosis was observed in $36 \%(9 / 25)$ of patients after a negative tau-PET, and in 50\% (1/2) after a negative amyloid-PET, with no difference between exams $\left(\chi^{2}=0, p=1.00\right)$. In patients with an etiological diagnosis of non-AD already supported by a first PET scan, a further change in diagnosis was observed in $100 \%$ (2/2) of patients after a positive tau-PET, and in 44\% (7/16) after a positive amyloid-PET, with no difference between exams $\left(\chi^{2}=0.56, p=0.453\right)$.

Table 3 provides detailed information on the diagnostic impact of the second PET scan in both diagnostic pathways. Most of the changes in diagnosis due to amyloid-PET or tau-PET presented as the second exam were observed in patients with discordant PET results (in 6 out of 8 , and 11 out of 12 cases respectively), or in patients whose baseline diagnoses were previously confirmed after the first scan (in 6 out of 8 , and 9 out of 12 cases respectively).

The only case in which the diagnosis changed after a consistent PET result was a patient with a T1 diagnosis of MCI not due to 
Table 1 Demographic and clinical features of participants

\begin{tabular}{|c|c|c|c|c|}
\hline Features & $\begin{array}{l}\text { Whole sample } \\
n=136\end{array}$ & $\begin{array}{l}\text { ARM1 } \\
n=68\end{array}$ & $\begin{array}{l}\text { ARM2 } \\
n=68\end{array}$ & $\begin{array}{l}\text { ARM1 vs ARM2 } \\
p \text { value }\end{array}$ \\
\hline Age & $74(70-78)$ & $73(70-78)$ & $74(70-78)$ & 0.442 \\
\hline Sex, male & $52 \%(71)$ & $51 \%(35)$ & $53 \%(36)$ & 1.000 \\
\hline Education, years & $14(11-18)[15]$ & $14(11-17)[8]$ & $15(12-18)[7]$ & 0.551 \\
\hline Symptom duration, years & $2(1-4)[26]$ & $2(1-4)[13]$ & $2(1-4)[13]$ & 0.968 \\
\hline MMSE & $27(24-28)[3]$ & $27(24-29)[1]$ & $27(24-28)[2]$ & 0.392 \\
\hline Cognitive stage & & & & 0.185 \\
\hline SCD & $18 \%(24)$ & $23 \%(16)$ & $12 \%(8)$ & \\
\hline MCI & $51 \%(69)$ & $48 \%(33)$ & $53 \%(36)$ & \\
\hline Dementia & $32 \%(43)$ & $28 \%(19)$ & $35 \%(24)$ & \\
\hline Etiological diagnosis, AD & $67 \%(91)$ & $63 \%(43)$ & $71 \%(48)$ & 0.466 \\
\hline Amyloid status, positive & $54 \%(73)$ & $54 \%(37)$ & $53 \%(36)$ & 1.000 \\
\hline Tau status, positive & $37 \%(50)$ & $38 \%(26)$ & $35 \%(24)$ & 0.859 \\
\hline \multicolumn{5}{|l|}{ MRI } \\
\hline MTA & $1.0(1.0-2.0)[33]$ & $1.0(1.0-2.0)[20]$ & $1.5(1.0-2.0)[13]$ & 0.094 \\
\hline Fazekas & $1.0(1.0-2.0)$ [39] & $1.0(1.0-2.0)[18]$ & $1.0(1.0-1.5)[21]$ & 0.175 \\
\hline Koedam & $1.0(1.0-1.5)[36]$ & $1.0(1.0-1.5)[22]$ & $1.0(1.0-1.5)[14]$ & 0.916 \\
\hline
\end{tabular}

$S C D$ : subjective cognitive decline, $M C I$ : mild cognitive impairment, $M T A$ : medial temporal atrophy

Numbers are median $\left(25^{\text {th }}-75^{\text {th }}\right.$ percentile) for continuous variables, and percentages (raw number) for the categorical variables. Square brackets [] indicate the number of missing values; reading example: MMSE score was not available for 3 patients, 1 of them in ARM1 and 2 in ARM2

Amyloid and tau status were assessed using amyloid-PET and tau-PET respectively

$\mathrm{AD}$ based on a negative amyloid-PET whose diagnosis changed to $\mathrm{AD}$ at $\mathrm{T} 2$ after a negative tau-PET scan (Braak stage $=\mathrm{I}-\mathrm{III})$ (see the "Discussion" section for further information on this case).

\section{Change in diagnostic confidence}

At T1, diagnostic confidence significantly increased by $18 \%$ after amyloid-PET $(p<0.001)$ and by $19 \%$ after tau-PET $(p<0.001)$ due to the first PET scan, with no difference between the two exams $(p=1.00)$. At T2, tau-PET further increased diagnostic confidence by $5 \%(p<0.001)$, and amyloid-PET by
$4 \%(p<0.001)$, with no difference in the final diagnostic confidence between the two pathways $(p=1.00)$ (Table 2 and Fig. 3 ).

\section{Change in diagnosis and diagnostic confidence in each cognitive stage group}

\section{SCD}

When presented as the first exam, the etiological diagnosis changed in $17 \%\left(4 / 24 ; \chi^{2}=0, p=1.00\right)$ of SCD patients after amyloid-PET, and in $17 \%\left(4 / 24 ; \chi^{2}=0.25\right.$,

Table 2 Overview of the primary outcomes (changes in diagnosis and changes in diagnostic confidence) in the whole sample and in the three cognitive stage groups (SCD, MCI, dementia)

\begin{tabular}{|c|c|c|c|c|c|c|c|c|c|}
\hline \multicolumn{2}{|c|}{ Primary outcomes } & \multicolumn{4}{|c|}{ Change in diagnosis ( $\%, p$ value $)$} & \multicolumn{4}{|c|}{ Change in diagnostic confidence ( $\%, p$ value) } \\
\hline & & $\begin{array}{l}\text { Whole sample } \\
n=136\end{array}$ & $\begin{array}{l}\mathrm{SCD} \\
n=24\end{array}$ & $\begin{array}{l}\text { MCI } \\
n=69\end{array}$ & $\begin{array}{l}\text { Dementia } \\
n=43\end{array}$ & $\begin{array}{l}\text { Whole sample } \\
n=136\end{array}$ & $\begin{array}{l}\mathrm{SCD} \\
n=24\end{array}$ & $\begin{array}{l}\text { MCI } \\
n=69\end{array}$ & $\begin{array}{l}\text { Dementia } \\
n=43\end{array}$ \\
\hline \multirow[t]{2}{*}{ First exam } & Amyloid-PET & $\begin{array}{l}28 \% \\
p=0.006\end{array}$ & $\begin{array}{l}17 \%, \\
p=1.00\end{array}$ & $\begin{array}{l}35 \% \\
p=0.008\end{array}$ & $\begin{array}{l}23 \% \\
p=0.343\end{array}$ & $\begin{array}{l}+18 \% \\
p<0.001\end{array}$ & $\begin{array}{l}+23 \% \\
p<0.001\end{array}$ & $\begin{array}{l}+19 \% \\
p<0.001\end{array}$ & $\begin{array}{l}+14 \% \\
p<0.001\end{array}$ \\
\hline & Tau-PET & $\begin{array}{l}28 \% \\
p<0.001\end{array}$ & $\begin{array}{l}17 \%, \\
p=0.617\end{array}$ & $\begin{array}{l}39 \% \\
p<0.001\end{array}$ & $\begin{array}{l}16 \% \\
p=0.023\end{array}$ & $\begin{array}{l}+19 \% \\
p<0.001\end{array}$ & $\begin{array}{l}+26 \% \\
p<0.001\end{array}$ & $\begin{array}{l}+22 \%, \\
p<0.001\end{array}$ & $\begin{array}{l}+10 \% \\
p<0.001\end{array}$ \\
\hline \multirow[t]{2}{*}{ Second exam } & Amyloid-PET & $\begin{array}{l}6 \% \\
p=0.077\end{array}$ & $0 \%$ & $\begin{array}{l}6 \% \\
p=0.134\end{array}$ & $\begin{array}{l}9 \% \\
p=0.617\end{array}$ & $\begin{array}{l}+4 \% \\
p<0.001\end{array}$ & $\begin{array}{l}+3 \% \\
p=1.00\end{array}$ & $\begin{array}{l}+3 \% \\
p=0.114\end{array}$ & $\begin{array}{l}+5 \% \\
p=0.005\end{array}$ \\
\hline & Tau-PET & $\begin{array}{l}9 \%, \\
p=0.149\end{array}$ & $0 \%$ & $\begin{array}{l}12 \% \\
p=0.289\end{array}$ & $\begin{array}{l}9 \%, \\
p=0.617\end{array}$ & $\begin{array}{l}+5 \% \\
p<0.001\end{array}$ & $\begin{array}{l}+6 \% \\
p=0.007\end{array}$ & $\begin{array}{l}+4 \%, \\
p=0.005\end{array}$ & $\begin{array}{l}+6 \% \\
p=0.001\end{array}$ \\
\hline
\end{tabular}

Bold $p$ values denote statistical significance $(p<0.05)$ 
Fig. 2 Change in diagnosis in the two diagnostic pathways (AMYTAU and TAU-AMY).
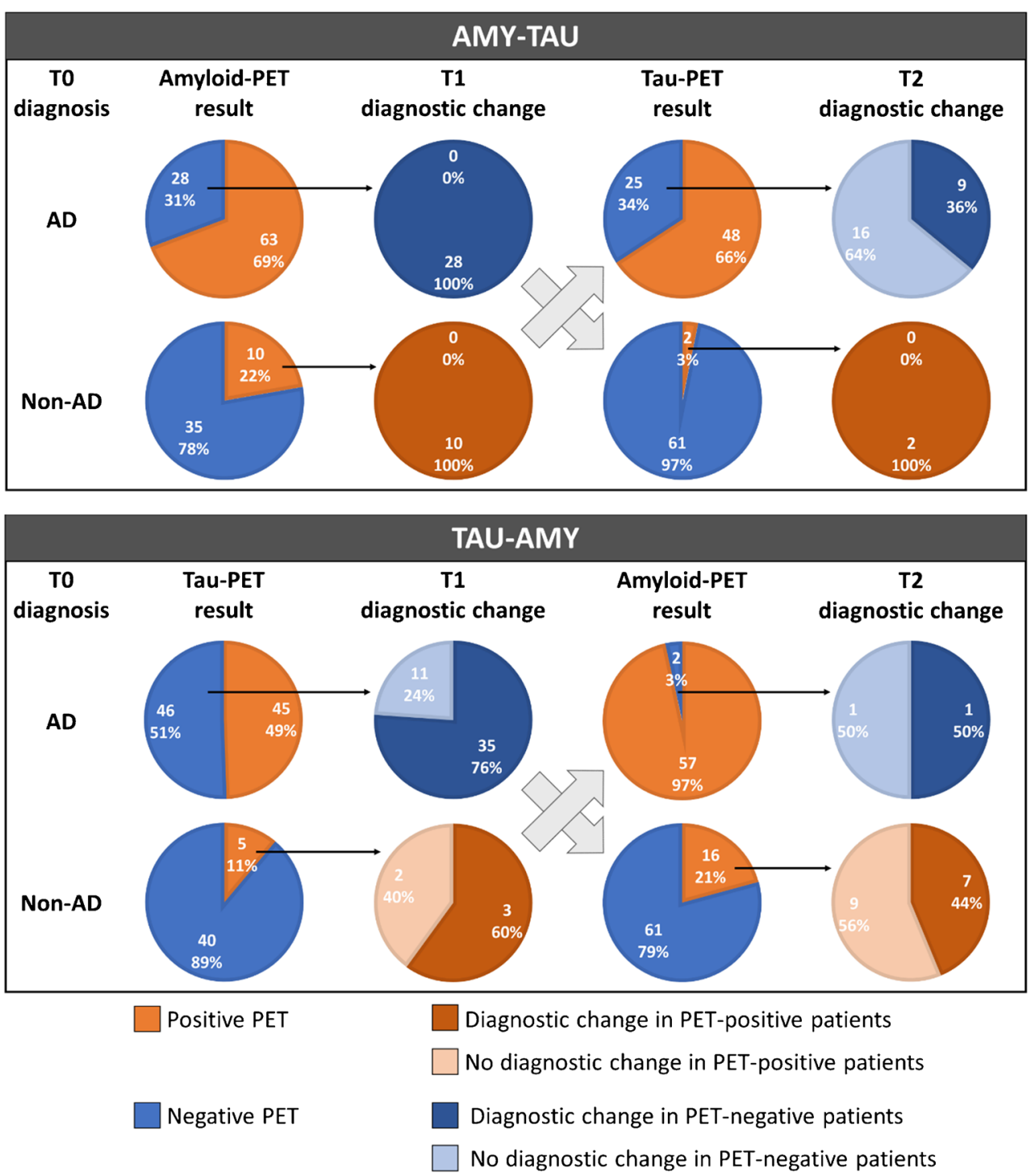

AMY-TAU: amyloid-PET presented as the first exam, followed by tau-PET. TAU-AMY: tau-PET presented as the first exam, followed by amyloid-PET.

T0: baseline assessment based on the clinical diagnostic workup. T1: first PET scan (either amyloid-PET or tau-PET according to the study design) added to the baseline assessment. T2: second PET scan added to the first examand the baseline assessment.

Reading example for the AMY-TAU pathway: at T0,91 patients had AD and, among them, $31 \%(28 / 91)$ had a negative amyloid-PET (first pie chart) resulting in a diagnostic change in $100 \%$ (28/28) of cases (second pie chart); at T1, 73 patients had AD and, among them, 34\% $(25 / 73)$ had a negative tau-PET (third pie chart) resulting in a diagnostic change in $36 \%$ $(9 / 25)$ of cases (fourth pie chart). $p=0.617)$ of SCD patients after tau-PET (Table 2), with no difference between the two exams $\left(\chi^{2}=0, p=1.00\right)$. The addition of a second PET scan resulted in no change in the etiological diagnosis of SCD patients both after amyloid-PET and tau-PET (Table 2). Figure S2 in the Supplementary Material illustrates how the diagnoses changed in SCD patients with PET results inconsistent with the previous etiological diagnosis.

When presented as the first exam, diagnostic confidence increased by $23 \%(p<0.001)$ after amyloid-PET, and by $26 \%$ $(p<0.001)$ after tau-PET (Table 2$)$, with no difference between the two exams $(p=1.00)$. Diagnostic confidence further 
Table 3 Detailed information on the diagnostic impact of the second PET scan in the two diagnostic pathways (AMY-TAU and TAU-AMY)

\begin{tabular}{|c|c|c|c|c|c|c|c|}
\hline $\begin{array}{l}\text { Diagnostic } \\
\text { pathway }\end{array}$ & $\begin{array}{l}\mathrm{N} \text { of } \\
\text { patients }\end{array}$ & $\begin{array}{l}\text { T0 } \\
\text { diagnosis }\end{array}$ & $\begin{array}{l}\text { First } \\
\text { PET scan }\end{array}$ & $\begin{array}{l}\mathrm{T} 1 \\
\text { diagnosis }\end{array}$ & $\begin{array}{l}\text { Second } \\
\text { PET scan }\end{array}$ & $\begin{array}{l}\mathrm{T} 2 \\
\text { diagnosis }\end{array}$ & Observations \\
\hline \multirow[t]{5}{*}{ AMY-TAU } & 8 & $\mathrm{AD}$ & $\mathrm{AMY}+$ & $\mathrm{AD}$ & TAU- & Non-AD & $\begin{array}{l}\text { - Discordant PET results } \\
\text { - Same diagnosis at T0 and T1 }\end{array}$ \\
\hline & 1 & Non-AD & $\mathrm{AMY}+$ & $\mathrm{AD}$ & TAU- & Non-AD & $\begin{array}{l}\text { - Discordant PET results } \\
\text { - T1 diagnosis changed after first PET scan }\end{array}$ \\
\hline & 1 & $\mathrm{AD}$ & AMY- & Non-AD & TAU+ & $\mathrm{AD}$ & $\begin{array}{l}\text { - Discordant PET results } \\
\text { - T1 diagnosis changed after first PET scan }\end{array}$ \\
\hline & 1 & Non-AD & AMY- & Non-AD & TAU+ & $\mathrm{AD}$ & $\begin{array}{l}\text { - Discordant PET results } \\
\text { - Same diagnosis at } \mathrm{T} 0 \text { and } \mathrm{T} 1\end{array}$ \\
\hline & $1^{*}$ & $\mathrm{AD}$ & AMY- & Non-AD & TAU- & $\mathrm{AD}$ & $\begin{array}{l}\text { - Concordant PET results } \\
\text { - T1 diagnosis changed after first PET scan }\end{array}$ \\
\hline \multirow[t]{4}{*}{ TAU-AMY } & 1 & $\mathrm{AD}$ & TAU- & $\mathrm{AD}$ & AMY- & Non-AD & $\begin{array}{l}\text { - Concordant PET results } \\
\text { - Same diagnosis at T0 and T1 }\end{array}$ \\
\hline & 4 & Non-AD & TAU- & Non-AD & $\mathrm{AMY}+$ & $\mathrm{AD}$ & $\begin{array}{l}\text { - Discordant PET results } \\
\text { - Same diagnosis at T0 and T1 }\end{array}$ \\
\hline & 2 & $\mathrm{AD}$ & TAU- & Non-AD & $\mathrm{AMY}+$ & $\mathrm{AD}$ & $\begin{array}{l}\text { - Discordant PET results } \\
\text { - T1 diagnosis changed after first PET scan }\end{array}$ \\
\hline & 1 & Non-AD & TAU+ & Non-AD & $\mathrm{AMY}+$ & $\mathrm{AD}$ & $\begin{array}{l}\text { - Concordant PET results } \\
\text { - Same diagnosis at T0 and T1 }\end{array}$ \\
\hline
\end{tabular}

$A M Y+/-$ : amyloid positive/negative, $T A U+/-$ : tau positive/negative

This table includes only patients who changed diagnosis after amyloid-PET $(n=8)$ or tau-PET $(n=12)$ presented as the second exam

*The only case in which the diagnosis changed after a consistent PET result was a patient with a T1 diagnosis of MCI not due to AD based on a negative amyloid-PET whose diagnosis changed to AD at T2 after a negative tau-PET scan (Braak stage $=$ I-III)

increased by $3 \%(p=1.00)$ after amyloid-PET and by $6 \%(p=$ $0.007)$ after tau-PET when presented as the second exams (Table 2), with no difference in the final diagnostic confidence between the two pathways $(p=1.00)$. Figure S3 in the Supplementary Material illustrates how the diagnostic confidence of SCD patients changed in the two diagnostic pathways.

\section{$\mathrm{MCl}$}

When presented as the first exam, the etiological diagnosis changed in $35 \%\left(24 / 69 ; \chi^{2}=7.0, p=0.008\right)$ of MCI patients after amyloid-PET, and in $39 \%\left(27 / 69 ; \chi^{2}=17.9, p<0.001\right)$ of MCI patients after tau-PET (Table 2), with no difference between the two exams $\left(\chi^{2}=0.1, p=0.724\right)$. The addition of a second PET scan resulted in a further change in the etiological diagnosis in $6 \%\left(4 / 69 ; \chi^{2}=2.2, p=0.134\right)$ of MCI patients after amyloid-PET, and $12 \%\left(8 / 69 ; \chi^{2}=1.1, p=0.289\right)$ of MCI patients after tau-PET (Table 2), with no difference between the two exams $\left(\chi^{2}=0.8, p=0.365\right)$. Figure S2 in the Supplementary Material illustrates how the diagnoses changed in MCI patients with PET results inconsistent with the previous etiological diagnosis.

When presented as the first exam, diagnostic confidence increased by $19 \%(p<0.001)$ after amyloid-PET, and by $22 \%(p<0.001)$ after tau-PET (Table 2$)$, with no difference between the two exams $(p=0.499)$. Diagnostic confidence further increased by $3 \%(p=0.114)$ after amyloid-PET and by $4 \%(p=0.005)$ after tau-PET when presented as the second exams (Table 2), with no difference in the final diagnostic confidence between the two pathways $(p=1.00)$. Figure S3 in the Supplementary Material illustrates how the diagnostic confidence of MCI patients changed in the two diagnostic pathways.

\section{Dementia}

When presented as the first exam, the etiological diagnosis changed in $23 \%\left(10 / 43 ; \chi^{2}=0.9, p=0.343\right)$ of dementia patients after amyloid-PET, and in $16 \%\left(7 / 43 ; \chi^{2}=5.14\right.$, $p=0.023$ ) of dementia patients after tau-PET (Table 2), with no difference between the two exams $\left(\chi^{2}=, p=\right.$ $0.588)$. The addition of a second PET scan resulted in a further change in the etiological diagnosis in $9 \%(4 / 43$; $\left.\chi^{2}=0.25, p=0.617\right)$ of dementia patients both after amyloid-PET and after tau-PET (Table 2), with no difference between the two exams $\left(\chi^{2}=0, p=1.00\right)$. Figure S2 in the Supplementary Material illustrates how the diagnoses changed in dementia patients with PET results inconsistent with the previous etiological diagnosis.

When presented as the first exam, diagnostic confidence increased by $14 \%(p<0.001)$ after amyloid-PET, and by $10 \%$ $(p<0.001)$ after tau-PET (Table 2$)$, with no difference between 


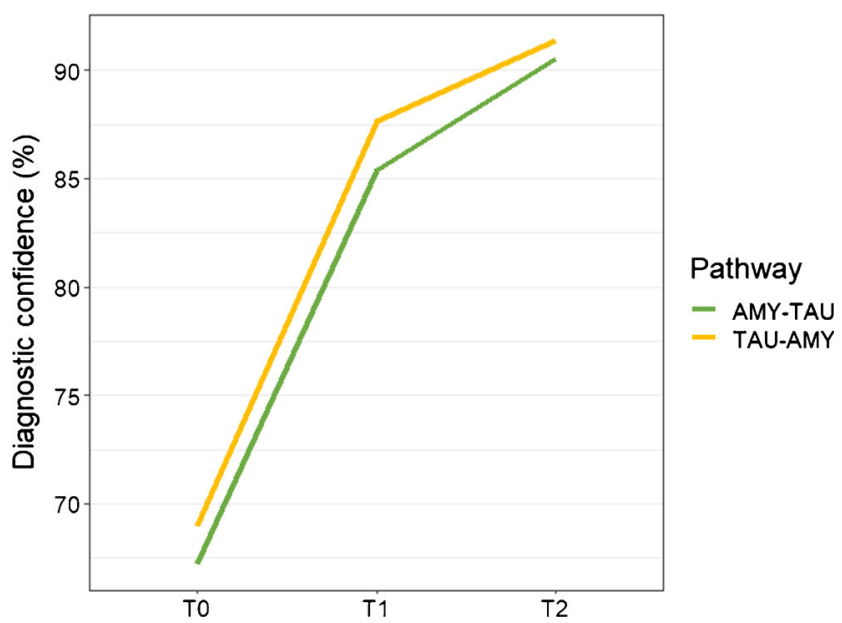

Values shown in this figure consist of the average of diagnostic confidences expressed by the two raters.

AMY-TAU: amyloid-PET presented as the first exam, followed by tau-PET. TAU-AMY tau-PET presented as the first exam, followed by amyloid-PET

T0: baseline assessment based on the clinical diagnostic workup. T1: first PET scan (either amyloid-PET or tau-PET according to the study design) added to the baseline assessment. T2: second PET scan added to the first examand the baseline assessment.

Fig. 3 Change in diagnostic confidence in the two diagnostic pathways (AMY-TAU and TAU-AMY)

the two exams $(p=0.807)$. Diagnostic confidence further increased by $5 \%(p=0.005)$ after amyloid-PET and by $6 \%(p=$ $0.001)$ after tau-PET when presented as the second exams (Table 2), with no difference in the final diagnostic confidence between the two pathways $(p=0.705)$. Figure S3 in the Supplementary Material illustrates how the diagnostic confidence of dementia patients changed in the two diagnostic pathways.

\section{Discordant amyloid-PET and tau-PET results}

The amyloid-PET and tau-PET results were reciprocally discordant in $20 \%(27 / 136)$ of cases, with 25 patients with positive amyloid-PET and negative tau-PET (Braak stage $=0$ in 14 patients, Braak stage $=$ I-III in 11 patients), and 2 cases having patients with negative amyloid-PET and positive tauPET (Braak stage $=\mathrm{V}$ in both cases). Among these discordant patients, the final (T2) diagnosis was consistent with the amyloid-PET result in 55\% (15/27) of cases for the first rater and in $67 \%(18 / 27)$ for the second rater, and with the tau-PET result in the remaining cases.

\section{Discussion}

In the present study, two independent raters assessed patients' clinical reports at three different timepoints, first based only on the routine diagnostic workup (clinical and neuropsychological assessment, MRI, other exams), and then based also on the availability of the first and second
PET scans in a randomized fashion. We observed that the impacts of amyloid-PET and tau-PET on diagnosis were overall similar: both PET scans induced significant and similar changes in diagnosis when presented as the first exam ( $28 \%$ due to amyloid-PET and $28 \%$ due to tau-PET), while the diagnostic change after the second exam was not significant for both PETs (6\% due to amyloid-PET and $9 \%$ due to tau-PET). This might be partially due to the high agreement between amyloid-PET and tau-PET (80\% in the present study), as compared to studies showing a moderate correlation between amyloid-PET and tau-PET in cognitively normal individuals [29]. Analyzing in detail the diagnostic impact of the second PET scan, most changes were observed in patients with discordant PET results or in patients whose baseline diagnoses were previously confirmed after the first scan, denoting that a second scan has a clinically significant impact (albeit not significant at group level) on some individuals with discordant biomarkers and even on those with a previous biomarker-based diagnosis.

All but one diagnostic change were due to PET results inconsistent with the previous etiological diagnosis. Interestingly, a negative amyloid-PET result had a stronger impact than a negative tau-PET when presented as the first exam $(100 \%$ vs $76 \%, p=0.014)$. The only case reclassified after a consistent PET scan was an amyloid-negative MCI patient who changed the etiological diagnosis from non-AD to AD after a tau-PET scan with Braak stage = I-III and an extensive temporal tau uptake, which one of the raters considered sufficient to have a diagnostic impact. This judgment was based on the evidence that subjects with Braak stage $=$ III have a neuropathologic diagnosis of AD in $63 \%$ of cases [30].

Significant and similar increases in diagnostic confidence due to amyloid-PET or tau-PET were observed when they were presented as the first or the second exams.

The above-described results were largely influenced by $\mathrm{MCI}$ patients, representing the largest cognitive stage group (i.e., $n=$ 69, vs 24 SCD and 43 dementia). Moreover, by qualitatively analyzing the individual cognitive stage groups, we observed that (i) MCI patients were those who benefitted the most from amyloid-PET and tau-PET in terms of change in diagnosis after the first exam (in 35\% and 39\% of cases respectively); (ii) SCD patients showed the highest increase in diagnostic confidence after the first exam $(+23 \%$ after amyloid-PET and $+26 \%$ after tau-PET), in spite of a very limited impact on diagnosis; and (iii) dementia patients showed a limited impact on diagnostic confidence after the first exam (+14\% after amyloid-PET and $+10 \%$ after tau-PET). We acknowledge that these subgroup analyses should be interpreted with caution, especially for SCD and dementia patients, due to small sample sizes.

Interestingly, the availability of at least one PET is enough to significantly increase the concordance between raters from fair $(71 \%$ concordance rate, unweighted $k=0.36)$ to good (87\% concordance rate, unweighted $k=0.74$ ). 
Several studies have already assessed the diagnostic value of amyloid-PET, supporting its potential utility in clinical practice [5], while no evidence is available on the diagnostic value of tau-PET or on their combination. To the best of our knowledge, this is the first study assessing the diagnostic value of tau-PET and comparing it with that of amyloid-PET in a memory clinic population. Our findings were partially discordant with our expectation of a higher diagnostic value of tau-PET. Indeed, studies on tau-PET with 18F-Flortaucipir showed that (i) Braak stages, representing tau load and topography, better correlate with clinical symptoms than amyloid load [29, 31, 32]; (ii) its positivity indicates both advanced amyloid and tau neuropathology [23]; (iii) it proved to be able to differentiate between amyloidpositive and amyloid-negative neurodegenerative diseases with high accuracy [33]; (iv) tau deposition is closely related to other markers of neuronal injury, e.g., FDGPET and gray matter atrophy [34-36]. On the other hand, amyloid-PET might be more sensitive than tau-PET to the early phases (amyloid is the first biomarker becoming abnormal during the AD course [37]), and, since it has been available for longer [4], it might be perceived by physicians as more reliable than tau-PET. In some cases, the raters attributed positive uptake on tau-PET to pathology other than ADrelated tau, as $18 \mathrm{~F}$-Flortaucipir binding had been reported in frontotemporal dementia forms [38]. This, together with the higher impact on the etiological diagnosis of a negative amyloid-PET (as compared to a negative tau-PET) as shown by our data, might have rebalanced the comparison.

This study fits into the phase 3 of the biomarker roadmap for the validation for Alzheimer's biomarkers [39] by providing pilot evidence on the comparative (secondary aim 2) and combined (secondary aim 3) diagnostic value of amyloid-PET and tau-PET.

\section{Limitations}

The main limitation of this study is its retrospective nature. The retrospective evaluation of clinical reports does not allow, by definition, the clinical observation of the patient, depriving physicians of a key element of the diagnostic process. As a consequence, the diagnostic classifications should be considered as diagnostic hypotheses within a research setting rather than as actual clinical diagnoses. For example, the two raters involved in this study made a baseline etiological diagnosis of $\mathrm{AD}$ in three SCD patients based on their clinical reports. The diagnostic confidence in these $\mathrm{AD}$ diagnoses was never higher than $55 \%$, corresponding to nearly the maximum uncertainty, meaning that the clinicians would not be confident in disclosing such diagnosis even if this is their diagnostic suspect. Nevertheless, the adopted study design allowed to compare and combine the diagnostic value of amyloid-PET and tau-
PET in a more structured way, which is not applicable in clinical practice (where physicians usually make a diagnosis only after the conclusion of the diagnostic workup, not after each exam). Moreover, it might be interesting to follow patients over time in order to assess the impact of amyloid-PET and tau-PET on a final longitudinal diagnosis (e.g., after 2, 5, or 10 years after baseline). Unfortunately, this longitudinal information was not available at the time of the present study. A prospective longitudinal study is needed to properly address this matter.

A second limitation is the dichotomization of the tau status. Indeed, while in clinical practice the amyloid-PET result is usually dichotomized based on established cut-offs, there are no clinically validated positivity cut-offs for tau-PET, the result of which can then be described on the basis of its topography. In the present study, significant tracer deposition in brain areas corresponding to Braak stages IV-VI was classified as tau positive in accordance with current knowledge on the cognitive impact of tau pathology [25] and on the detectability of tau pathology with 18F-Flortaucipir [26], and consistently with a recent study [27]. According to a different classification method, a positive tau-PET interpretation is consistent with Braak stages V-VI [23]. We underline that, according to this classification method, only $5 \%(7 / 136)$ of subjects, i.e., those who were classified as Braak stage $=I V$, would be considered as misclassified. Thus, we can conclude that the choice of the classification method had a limited influence on our results. Nevertheless, the raters did not receive only the dichotomized tau status but also the full tau-PET report with an estimate of the Braak stage. Thus, they could consider the whole picture and make their decisions accordingly. In fact, this explains why two patients changed diagnosis from non-AD to $\mathrm{AD}$ after a tau-PET Braak stage $=$ I-III, which was negative according to the study operationalization, but considered as already positive by one of the raters. Further research is needed to understand whether a dichotomous tau-PET result has a greater clinical value than a stage classification, and to validate visual assessment methods [7].

Finally, the study design prevents from drawing definitive conclusions on the diagnostic value of amyloid-PET and tauPET, which should ideally be assessed against a gold or reference standard informing about pathology, or evidence of progression with confirmation of diagnosis at follow-up examination. Even if this study provides pilot evidence on the clinical utility of amyloid-PET and tau-PET (biomarker roadmap phase 3 , secondary aims 2 and 3; and phase 4, primary aim 1), more studies on their analytical validity and clinical validity are still needed to validate their clinical use. Indeed, pathology-based studies are very rare but necessary to estimate the proportion of false-positive and false-negative cases against pathology, which will inform us about how many times clinicians will encounter a "true" $\mathrm{AD}$ (or non- $\mathrm{AD}$ ) patient with inconsistent/wrong tauPET or amyloid-PET (or both) scans. 


\section{Conclusion}

Amyloid-PET and tau-PET showed a significant and similar impact on etiological diagnosis and diagnostic confidence when presented as the first exam, although a negative amyloid-PET showed a higher impact on etiological diagnosis than a negative tau-PET. The impact of both exams was still significant in terms of improved diagnostic confidence when added as the second PET exam.

Supplementary Information The online version contains supplementary material available at https://doi.org/10.1007/s00259-021-05246-x.

Acknowledgements We thank Avid Radiopharmaceuticals for providing access to the 18F-Flortaucipir tracer; Prof. Roger Schibli's group at ETH, Zurich, for tracer synthesis and quality control; and the Clinical Research Center, University Hospital and Faculty of Medicine, Geneva, for its contribution.

Funding Open Access funding provided by Université de Genève. PET scans were performed within research projects funded by the Swiss National Science Foundation (SNSF, project numbers: 320030 169876 and 320030 185028) and the EU-EFPIA Innovative Medicines Initiatives 2 Joint Undertaking (IMI 2 JU) Amyloid Imaging to Prevent Alzheimer's Disease (AMYPAD, grant agreement number: 115952). Camilla Caprioglio was supported by the EU-EFPIA Innovative Medicines Initiatives 2 Joint Undertaking (IMI 2 JU) Amyloid Imaging to Prevent Alzheimer's Disease (AMYPAD, grant agreement number: 115952). Kelly Ceyzériat was supported by the Velux Foundation (grant agreement number: 1123). Giovanni B. Frisoni received funding from the EU-EFPIA Innovative Medicines Initiative Joint Undertaking (IMI JU) European Prevention of Alzheimer's Dementia Consortium (EPAD, grant agreement number: 115736) and the EU-EFPIA Innovative Medicines Initiatives 2 Joint Undertaking (IMI 2 JU) Amyloid Imaging to Prevent Alzheimer's Disease (AMYPAD, grant agreement number: 115952). Valentina Garibotto was supported by the Swiss National Science Foundation (projects 320030_169876, 320030_185028, and IZSEZ0 188355) and by the Velux Foundation (project 11 123 ).

\section{Declarations}

Ethical approval All procedures performed in studies involving human participants were in accordance with the ethical standards of the institutional and/or national research committee and with the 1964 Helsinki declaration and its later amendments or comparable ethical standards. The study was approved by the local ethic committee (PB 2016-01346).

Informed consent Each subject provided a voluntary written informed consent for the participation in the study.

Conflict of interest Daniele Altomare declares that he has no conflict of interest.

Camilla Caprioglio declares that she has no conflict of interest.

Frédéric Assal declares that he has no conflict of interest.

Gilles Allali declares that he has no conflict of interest.

Aline Mendes declares that she has no conflict of interest.

Federica Ribaldi declares that she has no conflict of interest.

Kelly Ceyzeriat declares that she has no conflict of interest.

Marta Martins declares that she has no conflict of interest.

Szymon Tomczyk declares that he has no conflict of interest.
Sara Stampacchia declares that she has no conflict of interest. Alessandra Dodich declares that she has no conflict of interest. Marina Boccardi declares that she has no conflict of interest.

Christian Chicherio declares that he has no conflict of interest.

Giovanni B. Frisoni reports grants from Alzheimer Forum Suisse, Académie Suisse des Sciences Médicales, Avid Radiopharmaceuticals, Biogen, GE International, Guerbert, Association Suisse pour la Recherche sur l'Alzheimer, IXICO, Merz Pharma, Nestlé, Novartis, Piramal, Roche, Siemens, Teva Pharmaceutical Industries, Vifor Pharma, and Alzheimer's Association; he has received personal fees from AstraZeneca, Avid Radiopharmaceuticals, Elan Pharmaceuticals, GE International, Lundbeck, Pfizer, and TauRx Therapeutics.

Valentina Garibotto received financial support for research through her institution from Siemens Healthineers, GE Healthcare, Life Molecular Imaging, Cerveau Technologies, Roche, Merck.

Disclaimer This communication reflects the views of the authors and neither IMI nor the European Union and EFPIA are liable for any use that may be made of the information contained herein.

Open Access This article is licensed under a Creative Commons Attribution 4.0 International License, which permits use, sharing, adaptation, distribution and reproduction in any medium or format, as long as you give appropriate credit to the original author(s) and the source, provide a link to the Creative Commons licence, and indicate if changes were made. The images or other third party material in this article are included in the article's Creative Commons licence, unless indicated otherwise in a credit line to the material. If material is not included in the article's Creative Commons licence and your intended use is not permitted by statutory regulation or exceeds the permitted use, you will need to obtain permission directly from the copyright holder. To view a copy of this licence, visit http://creativecommons.org/licenses/by/4.0/.

\section{References}

1. Villemagne VL, Doré V, Burnham SC, Masters CL, Rowe CC. Imaging tau and amyloid- $\beta$ proteinopathies in Alzheimer disease and other conditions. Nat Rev Neurol. 2018;14:225-36. https://doi. org/10.1038/nrneurol.2018.9.

2. Dubois B, Hampel H, Feldman HH, Scheltens P, Aisen P, Andrieu $S$, et al. Preclinical Alzheimer's disease: definition, natural history, and diagnostic criteria. Alzheimers Dement. 2016;12:292-323. https://doi.org/10.1016/j.jalz.2016.02.002.

3. Jack CR, Bennett DA, Blennow K, Carrillo MC, Dunn B, Haeberlein SB, et al. NIA-AA research framework: toward a biological definition of Alzheimer's disease. Alzheimers Dement. 2018;14:535-62. https://doi.org/10.1016/j.jalz.2018.02.018.

4. Klunk WE, Engler H, Nordberg A, Wang Y, Blomqvist G, Holt DP, et al. Imaging brain amyloid in Alzheimer's disease with Pittsburgh compound-B. Ann Neurol. 2004;55:306-19. https:// doi.org/10.1002/ana.20009.

5. Barthel H, Sabri O. Clinical use and utility of amyloid imaging. J Nucl Med. 2017;58:1711-7. https://doi.org/10.2967/jnumed.116. 185017.

6. Chien DT, Bahri S, Szardenings AK, Walsh JC, Mu F, Su MY, et al. Early clinical PET imaging results with the novel PHF-tau radioligand [F-18]-T807. J Alzheimers Dis. 2013;34:457-68. https://doi.org/10.3233/JAD-122059.

7. Wolters EE, A. Dodich A, Boccardi M, Corre j, Drzezga A, Hansson $\mathrm{O}$ et al. Clinical validity of increased cortical uptake of [18F]flortaucipir on PET as a biomarker for Alzheimer's disease in the context of a structured 5-phase biomarker development 
framework. Eur J Nucl Med Mol Imaging. 2021. https://doi.org/ 10.1007/s00259-020-05118-w

8. FDA Approves First Drug to Image Tau Pathology in Patients Being Evaluated for Alzheimer's Disease | FDA n.d. https://www. fda.gov/news-events/press-announcements/fda-approves-firstdrug-image-tau-pathology-patients-being-evaluated-alzheimersdisease (accessed September 7, 2020).

9. Ramusino MC, Garibotto V, Bacchin R, Altomare D, Dodich A, Assal F, et al. Incremental value of amyloid-PET versus CSF in the diagnosis of Alzheimer's disease. Eur J Nucl Med Mol Imaging. 2020;47:270-80. https://doi.org/10.1007/s00259-019-04466-6.

10. Jessen F, Amariglio RE, Buckley RF, van der Flier WM, Han Y, Molinuevo JL, et al. The characterisation of subjective cognitive decline. Lancet Neurol. 2020;19:271-8. https://doi.org/10.1016/ S1474-4422(19)30368-0.

11. Albert MS, DeKosky ST, Dickson D, Dubois B, Feldman HH, Fox $\mathrm{NC}$, et al. The diagnosis of mild cognitive impairment due to Alzheimer's disease: recommendations from the National Institute on Aging-Alzheimer's Association workgroups on diagnostic guidelines for Alzheimer's disease. Alzheimers Dement. 2011;7: 270-9. https://doi.org/10.1016/j.jalz.2011.03.008.

12. McKhann GM, Knopman DS, Chertkow H, Hyman BT, Jack CR, Kawas CH, et al. The diagnosis of dementia due to Alzheimer's disease: recommendations from the National Institute on AgingAlzheimer's Association workgroups on diagnostic guidelines for Alzheimer's disease. Alzheimers Dement. 2011;7:263-9. https:// doi.org/10.1016/j.jalz.2011.03.005.

13. Chui HC, Mack W, Jackson JE, Mungas D, Reed BR, Tinklenberg $\mathrm{J}$, et al. Clinical criteria for the diagnosis of vascular dementia: a multicenter study of comparability and interrater reliability. Arch Neurol. 2000;57:191-6. https://doi.org/10.1001/archneur.57.2.191.

14. Román GC, Tatemichi TK, Erkinjuntti T, Cummings JL, Masdeu $\mathrm{JC}$, Garcia JH, et al. Vascular dementia: diagnostic criteria for research studies: report of the ninds-airen international workshop*. Neurology. 1993;43:250-60. https://doi.org/10.1212/wnl.43.2.250.

15. Erkinjuntti T, Inzitari D, Pantoni L, Wallin A, Scheltens P, Rockwood K, et al. Research criteria for subcortical vascular dementia in clinical trials. J Neural Transm Suppl. 2000;59:23-30. https://doi.org/10.1007/978-3-7091-6781-6_4.

16. Rascovsky K, Hodges JR, Knopman D, Mendez MF, Kramer JH, Neuhaus J, et al. Sensitivity of revised diagnostic criteria for the behavioural variant of frontotemporal dementia. Brain. 2011;134: 2456-77. https://doi.org/10.1093/brain/awr179.

17. McKeith IG, Dickson DW, Lowe J, Emre M, O'Brien JT, Feldman $\mathrm{H}$, et al. Diagnosis and management of dementia with Lewy bodies: third report of the DLB consortium. Neurology. 2005;65:1863-72. https://doi.org/10.1212/01.wnl.0000187889.17253.b1.

18. Jack CR, Knopman DS, Weigand SD, Wiste HJ, Vemuri P, Lowe $\mathrm{V}$, et al. An operational approach to National Institute on Aging-Alzheimer's Association criteria for preclinical Alzheimer disease. Ann Neurol. 2012;71:765-75. https://doi. org/10.1002/ana.22628.

19. Jack CR, Knopman DS, Chételat G, Dickson D, Fagan AM, Frisoni $\mathrm{GB}$, et al. Suspected non-Alzheimer disease pathophysiologyconcept and controversy. Nat Rev Neurol. 2016;12:117-24. https://doi.org/10.1038/nrneurol.2015.251.

20. Shcherbinin S, Schwarz AJ, Joshi A, Navitsky M, Flitter M, Shankle WR, et al. Kinetics of the tau PET tracer 18F-AV-1451 (T807) in subjects with normal cognitive function, mild cognitive impairment, and Alzheimer disease. J Nucl Med. 2016;57:153542. https://doi.org/10.2967/jnumed.115.170027.

21. Vizamyl - European Medicines Agency - Summary of Product Characteristics n.d. https://www.ema.europa.eu/en/documents/ product-information/vizamyl-epar-product-information en.pdf (accessed October 13, 2020).
22. Amyvid - European Medicines Agency - Summary of Product Characteristics n.d. https://www.ema.europa.eu/en/documents/ product-information/amyvid-epar-product-information_en.pdf (accessed October 13, 2020).

23. Fleisher AS, Pontecorvo MJ, Devous MD, Lu M, Arora AK, Truocchio SP, et al. Positron emission tomography imaging with $[18 \mathrm{~F}]$ flortaucipir and postmortem assessment of Alzheimer disease neuropathologic changes. JAMA Neurol. 2020. https://doi.org/10. 1001/jamaneurol.2020.0528.

24. Dodich A, Rochat A-S, Mainta I, Noirot C, Andryszak P, Rakotomiaramanana B, et al. Validation of a visual assessment strategy for 18F-Flortaucipir PET. EANM. 2019;19.

25. Gold G, Bouras C, Kövari E, Canuto A, Glaría BG, Malky A, et al. Clinical validity of Braak neuropathological staging in the oldestold. Acta Neuropathol. 2000;99:579-82. https://doi.org/10.1007/ s004010051163.

26. Lowe VJ, Lundt ES, Albertson SM, Min HK, Fang P, Przybelski SA, et al. Tau-positron emission tomography correlates with neuropathology findings. Alzheimers Dement. 2020;16:561-71. https://doi.org/10.1016/j.jalz.2019.09.079.

27. Sonni I, Lesman Segev OH, Baker SL, Iaccarino L, Korman D, Rabinovici GD, et al. Evaluation of a visual interpretation method for tau-PET with 18 F-flortaucipir. Alzheimer's Dement Diagnosis, Assess Dis Monit. 2020;12. https://doi.org/10. 1002/dad2.12133.

28. Frisoni GB, Barkhof F, Altomare D, Berkhof J, Boccardi M, Canzoneri E, et al. AMYPAD diagnostic and patient management study: rationale and design. Alzheimers Dement. 2019;15:388-99. https://doi.org/10.1016/j.jalz.2018.09.003.

29. Aschenbrenner AJ, Gordon BA, Benzinger TLS, Morris JC, Hassenstab JJ. Influence of tau PET, amyloid PET, and hippocampal volume on cognition in Alzheimer disease. Neurology. 2018;91:e859-66. https://doi.org/10.1212/WNL. 0000000000006075 .

30. Mufson EJ, Malek-Ahmadi M, Snyder N, Ausdemore J, Chen K, Perez SE. Braak stage and trajectory of cognitive decline in noncognitively impaired elders. Neurobiol Aging. 2016;43:10110. https://doi.org/10.1016/j.neurobiolaging.2016.03.003.

31. Ossenkoppele R, Smith R, Ohlsson T, Strandberg O, Mattsson N, Insel PS, et al. Associations between tau, $\mathrm{A} \beta$, and cortical thickness with cognition in Alzheimer disease. Neurology. 2019;92:e601-12. https://doi.org/10.1212/WNL.0000000000006875.

32. Bennett DA, Schneider JA, Wilson RS, Bienias JL, Arnold SE. Neurofibrillary tangles mediate the association of amyloid load with clinical Alzheimer disease and level of cognitive function. Arch Neurol. 2004;61:378-84. https://doi.org/10.1001/archneur. 61.3.378.

33. Hammes J, Bischof G, Bohn K, Onur O, Schneider A, Fliessbach $\mathrm{K}$, et al. One stop shop: flortaucipir PET differentiates amyloid positive and negative forms of neurodegenerative diseases. J Nucl Med. 2020. https://doi.org/10.2967/jnumed.120.244061.

34. Xia C, Makaretz SJ, Caso C, McGinnis S, Gomperts SN, Sepulcre $\mathrm{J}$, et al. Association of in vivo [18F]AV-1451 tau PET imaging results with cortical atrophy and symptoms in typical and atypical Alzheimer disease. JAMA Neurol. 2017;74:427-36. https://doi. org/10.1001/jamaneurol.2016.5755.

35. Van Eimeren T, Bischof GN, Drzezga A. Is tau imaging more than just upside-down 18F-FDG imaging? J Nucl Med. 2017;58:13579. https://doi.org/10.2967/jnumed.117.190082.

36. Chiotis K, Saint-Aubert L, Rodriguez-Vieitez E, Leuzy A, Almkvist O, Savitcheva I, et al. Longitudinal changes of tau PET imaging in relation to hypometabolism in prodromal and Alzheimer's disease dementia. Mol Psychiatry. 2018;23:1666-73. https://doi.org/10.1038/mp.2017.108.

37. Jack CR, Knopman DS, Jagust WJ, Petersen RC, Weiner MW, Aisen PS, et al. Tracking pathophysiological processes in 
Alzheimer's disease: an updated hypothetical model of dynamic biomarkers. Lancet Neurol. 2013;12:207-16. https://doi.org/10. 1016/S1474-4422(12)70291-0.

38. Tsai RM, Bejanin A, Lesman-Segev O, Lajoie R, Visani A, Bourakova V, et al. 18F-flortaucipir (AV-1451) tau PET in frontotemporal dementia syndromes. Alzheimers Res Ther. 2019;11. https://doi.org/10.1186/s13195-019-0470-7.

39. Boccardi M, Dodich A, Albanese E, Gayet-Ageron A, Walter M, Rabinovici GD, et al. The biomarker roadmap for the validation for
Alzheimer's biomarkers: Methodological update for biomarkers of tauopathy. Alzheimers Dement. 2020. https://doi.org/10.1002/alz. 039063.

Publisher's note Springer Nature remains neutral with regard to jurisdictional claims in published maps and institutional affiliations. 